\title{
Performance Measure Modeling Of Routing Protocols In MANET
}

\author{
Anmol Preet Kaur ${ }^{1}$, Nitika Chowdhary ${ }^{2}$ and Jyoteesh Malhotra ${ }^{3}$ \\ ${ }^{1}$ ECE Department, ${ }^{2}$ CSE Department, ${ }^{3}$ ECE Department \\ Ianmolmattewal58@yahoo.com, ${ }^{2}$ nitu.expert@gmail.com, ${ }^{3}$ jyoteesh@gmail.com
}

\begin{abstract}
Mobile ad-hoc networks (MANETs) are recently attracting a lot of interest due to their widely available wireless devices and low communication cost. MANET comprises a set of wireless communicating devices able to connect instantaneously without any preexisting base station. Due to a limited transmission range of mobile devices, they need multi-hop routing for communication. In MANET nodes can change topology in an unpredictable manner so they need an efficient routing protocol which can handle the changing topology. However, MANET suffers from many challenges, including decentralization, low memory device, infrastructure less, bandwidth and limited battery reservoir. Different modes of the nodes like active-communication mode and non-activecommunication mode consume available energy of a node. This paper presents an energy measurement model which shows the remaining energy level in each mobile node after some activity. The parameters required for designing energy measurement model consist of initialEnergy, txPower, rxPower, and idlePower. This paper also evaluates the performance measure metrics of two routing protocols such as Ad hoc On Demand Distance Vector (AODV) and Destination Sequenced Distance Vector (DSDV). This paper analyzes these routing protocols by simulation tested in network simulator (NS2). The performance will be studied based on Throughput, Packet loss, Routing delay, Cumulative sum of dropped packets, Calculate energy consumption and remaining energy at each node.
\end{abstract}

Keywords: Decentralization, on- the fly network, energy model, cumulative sum of dropped packets

\section{Introduction}

With the rapid growth in wireless communication and mobile devices in recent years, it has become necessary to stay online all the time. Ad-hoc networks are short distance networks and each node in the network connected by wireless links. They are created when device uses the same routing protocol. Ad-hoc network is of LAN type, shortly live, on-the fly network also called mesh network. All mobile nodes of this network behave as host and as routers. As a host, nodes function as a transmitter and a receiver in the network and as a hop, nodes act as intermediate bridges between the transmitter and receiver giving store-and-forward services to all the neighboring nodes in the network. MANET is a collection of wireless mobile nodes that dynamically built a temporary network without any central control base station. Such devices can communicate with other device that is immediately within their radio range. If wireless devices are out of their transmission ranges, other mobile node present between them can forward their messages. Thus, communication between these nodes is established by multi hopping. Any new nodes can join/leave the topology at any time or turned on/off without notifying other nodes. This leads to changes in dynamic topology which is composed of variable bandwidth in wireless links. Due to the randomly movable wireless node around the whole network, each node needs to be equipped with the capability of an autonomous system, RF transceiver as it maintains connectivity and reach ability. The RF transceiver 
has limited source of energy in the form of battery. There are some reasons for early depletion of battery power in MANET is described below:

- Decentralize network: This creates the problem of medium access control by available nodes, as mobile nodes may compete simultaneously for medium access. All the packets will be collide, when two or more nodes transmit packet at the similar time and loses their energy.

- Overhearing: When a node receives packets that are not sent for it, then it said to be in overhearing mode. The unnecessarily receiving such packets will consume a considerable amount of energy.

- Control packet overhead: To enable data transmission between sender and receiver, the least number of control packets overhead should be used. For transmitting, generating and receiving these packets consumes energy.

- Idle listening: This happens, when a node listens to an idle channel to get probable traffic. This is the largest source of energy wastage.

- Interference: A node located between transmission range and interference range receives a packet but cannot decode it. The receiving and processing the packets consume energy.

- Battery constraint: The mobile hosts in ad-hoc networks are usually battery powered so choosing an energy efficient route is important. Early battery depletion of a wireless node not only affect the node, but also its capacity to forward packets on behalf of others and reduce the span of network lifetime.

The organization of paper is defined as follows: Section 2 gives the brief discussion of ad-hoc routing protocols, Section 3 defines the network simulation setups and their results, Section 4 gives the comparative analysis of two routing protocols, and Section 5 describes the conclusion and future scope of the paper.

\section{Background}

In this section briefly describes the proposed routing protocols in mobile ad-hoc networks.

\subsection{Ad- Hoc On Demand Distance Vector Routing Protocol (AODV)}

AODV is a reactive routing protocol using on demand route discovery process in adhoc networking. It has the benefit of low routing overhead and significant high delay while route discovering. The routing overheads which contain control messages are flooded to the complete network. At any time the sender node wants to send data to the receiver, if the source node doesn't have route information in its own routing table, sender initiates the route discovery process to destination by broadcasting the RREQ (route request) packets to its neighbor node. The RREQ packet has broadcast ID, the address of the sender and receiver, destination sequence numbers and number of hops included in the route. The sequence number ensures that routes are free from loops and has the fresh route. The nodes which receive the RREQ packet in turn again broadcast the request packet to its own neighbors. If the neighbor node will be the intended receiver node, then the node will send a unicast route replay (RREP) message, along the reverse path made from the sender of RREQ packet. Route Request (RREQ) consist the last known sequence number for the intended receiver. The intermediate nodes that forward the RREP packet to their neighbor, also records the coming hops in a route to the receiver. When the sequence number of the path is larger than or equal to the sequence number comprised in the current RREQ packet, then the intermediate node that receives the RREQ packet replies only. There is a predefined lifetime value for every reverse path entry in the routing table and it is deleted after exceeding timeout interval and routes dropped from the table. But if the similar routes are used, then the lifetime duration is updated so that those routes are not broken. The intermediate node periodically sends HELLO message to 
each other. If the nodes do not send replies due to link breakages within their route active time, then Route ERROR (RERR) message is broadcast to notify the other nodes.

\subsection{Destination Sequenced Distance-Vector Routing Protocol (DSDV)}

DSDV is a proactive routing protocol based on the traditional Bellman-Ford routing algorithm. The improvements made to the Bellman-Ford mechanism include freedom from loops using destination sequence number in the routing tables. Every mobile node maintains a routing table that records all available receiver nodes, the number of available hops to each the destination and each route is defined by the sequence number originated and incremented by the receiver node. Each station periodically sends their updated routing tables to their neighbors in the network. The sequence number is used to find outdated routes from the fresh routes and nodes with similar sequence number routes with smaller hop count are used to select the shortest path. Each node in the network is an event driven as it knows the state and topology of the entire network by transmitting the routing table when there is a change in the topology from last update. The routes are selected by a metric of least delay, good signal strength, minimum no of hops, etc. DSDV cannot find route reliable for longer duration when there is high mobility in the network. In DSDV, when a link break is detected at node $\mathrm{N}$ :

- The broken links defined by the worst value $\propto$

- The route with broken link in any hop is indicated by $\propto$.

- $\quad$ The nodes are quickly informed by flooding $\propto$ to their neighbors.

The routing overhead increases as the number of nodes increases. To solve this problem, broadcasting is limited by "full dump" and "incremental packets". Full dump includes all available route information and infrequently transmitted while there is not much movement, whereas in an incremental packet carries smaller message information that was updated since the last full dump.

\subsection{Performance Metrics}

- Energy Consumption (EC): The level of energy spent by node to transmit and receive the number of data packets and is defined below:

$\mathrm{EC}=\mathrm{A}$ node initial energy - Energy required for sending data packets

EC is measured in Joules.

- Residual energy: The remaining energy in a node after some activity.

- Average Energy Consumption (AEC): It is an average amount of energy consumed by each node to transmit data packets from sender to receiver in multi hop fashion and is defined below:

$$
\mathrm{AEC}=\frac{\text { The total energy consumed }}{\text { Total no. of available nodes }}
$$

AEC is measured in Joules.

- $\quad$ Packet Delivery Fraction (PDF): The ratio of total number of data packets successfully delivered to the receiver to the total number of data packets sends by sender and is defined below:

$$
\mathrm{PDF}=\frac{\text { Successfully received data packets }}{\text { Total no of sending packets }} \times 100 \%
$$


- Throughput: The ratio of total number of packets received by the destination for the time required to travel packet from sender to receiver and is given below:

$$
\mathrm{T}=\frac{\text { Total no of receiving packets from node }}{\text { Data transmission period }} * 8
$$

$\mathrm{T}$ is measured in bps.

- $\quad$ End-to-End Delay (E2ED): It is the delay of receiving packets between sender and receiver nodes. It is measured by summing of delays of number of hops involved between sender and receiver.

- Packet Loss (PL): It is defined as no of packets generated by a source node to the number of packets received by destination node and it is defined as

$\mathrm{PL}=$ Total no. of generating data packets - Total no. of receiving data packets

\section{Simulation Setup and Results}

The energy model was performed simulations using Network Simulator 2 (NS-2), particularly popular in the field of wireless ad hoc networking. The Random Waypoint Model is used as mobility model. The CBR (continuous bit rate) are traffic generator, the number of data connection are 5, max. Size of the data packet is 512 bytes and data sending rate is 4 packet/second. The nodes are spread randomly over the network area of $2000 \mathrm{~m} \times 2000 \mathrm{~m}$. During the simulation, each node has a random position at the start and chosen randomly destination position. The total time period of the simulation is 60 seconds and the maximum transmission range of nodes is $250 \mathrm{~m}$. The interface queue length is 100 packet drop-tail priority queues.

Table 1. Simulation Parameters

\begin{tabular}{|c|c|}
\hline Parameters & Values \\
\hline Channel & Wireless \\
\hline Network interface & WirelessPhy \\
\hline Area & $2000 \mathrm{~m}^{*} 2000 \mathrm{~m}$ \\
\hline Simulation time & $60 \mathrm{sec}$ \\
\hline Maximum packet in Queue & 100 \\
\hline Packet size & 512 bytes \\
\hline Data type & CBR \\
\hline No of nodes & 30 \\
\hline No of connections & 5 \\
\hline Antenna & Omni-Antenna \\
\hline Protocol used & AODV, DSDV \\
\hline Propagation & Two Ray Ground \\
\hline MAC type & Mac $802-11$ \\
\hline Transmission range & $250 \mathrm{~m}$ \\
\hline Initial energy & 200 joules \\
\hline Rx Power & 0.9 joules \\
\hline Tx Power & 1.4 joules \\
\hline Idle Power & 0.73 joules \\
\hline
\end{tabular}




\subsection{Simulation Results}

The simulation result shows the several differences in the two routing protocols. They are discussed in following subsections.

Table 2. Graph Parameters

\begin{tabular}{|l|l|l|l|}
\hline $\begin{array}{c}\text { Source node }- \\
\text { Sink node }\end{array}$ & $\begin{array}{c}\text { Time to start } \\
\text { transmission }\end{array}$ & $\begin{array}{c}\text { Color of } \\
\text { graph }\end{array}$ & $\begin{array}{c}\text { Time to end } \\
\text { simulation }\end{array}$ \\
\hline N0 - N1 & $10 \mathrm{sec}$ & RED & $60 \mathrm{sec}$ \\
\hline N5 - N6 & $15 \mathrm{sec}$ & BLUE & $60 \mathrm{sec}$ \\
\hline N3 - N6 & $18 \mathrm{sec}$ & YELLOW & $60 \mathrm{sec}$ \\
\hline
\end{tabular}

3.1.1. Throughput: The throughput is measured in bandwidth of $10^{*-3} \mathrm{~Hz}$ w.r.t to time (sec).

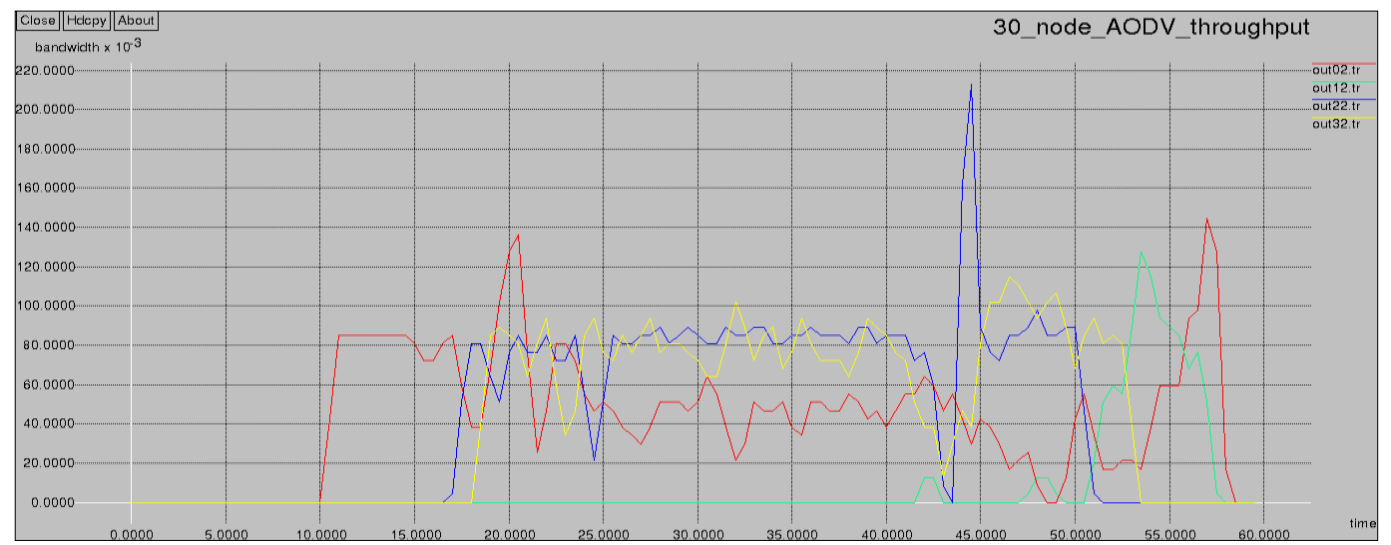

Figure 1. AODV Throughput

Table 3. AODV Throughput Comparison Between Three Sources - Sink Nodes

\begin{tabular}{|l|l|l|l|}
\hline $\begin{array}{c}\text { Time in } \\
\text { sec }\end{array}$ & N0 - N1 & N5 - N6 & N3 - N6 \\
\hline 10.5 & 0.04 & 0 & 0 \\
\hline 17 & 0.08 & 0.004 & 0 \\
\hline 18.5 & 0.03 & 0.08 & 0.03 \\
\hline 20.5 & 0.13 & 0.08 & 0.08 \\
\hline 24.5 & 0.04 & 0.02 & 0.09 \\
\hline 34.5 & 0.05 & 0.08 & 0.06 \\
\hline 43.5 & 0.05 & 0 & 0.02 \\
\hline 46.5 & 0.01 & 0.07 & 0.1 \\
\hline 53 & 0.017 & 0 & 0 \\
\hline
\end{tabular}

Table 3 shows the output of AODV throughput. Figure 1 shows the graph, in this $\mathrm{x}$ axis shows the time and y-axis shows the throughput. The graph is plotted between three connections of nodes. Each node starts transmission at different times having UDP traffic agent with CBR traffic source. Below these time instances the throughput remains zero. The graph shows node 0 starts transmission at $10.5 \mathrm{sec}$, node 5 starts at $17 \mathrm{sec}$, and node 3 at $0.03 \mathrm{sec}$. The maximum value of AODV throughput is 0.1 . 


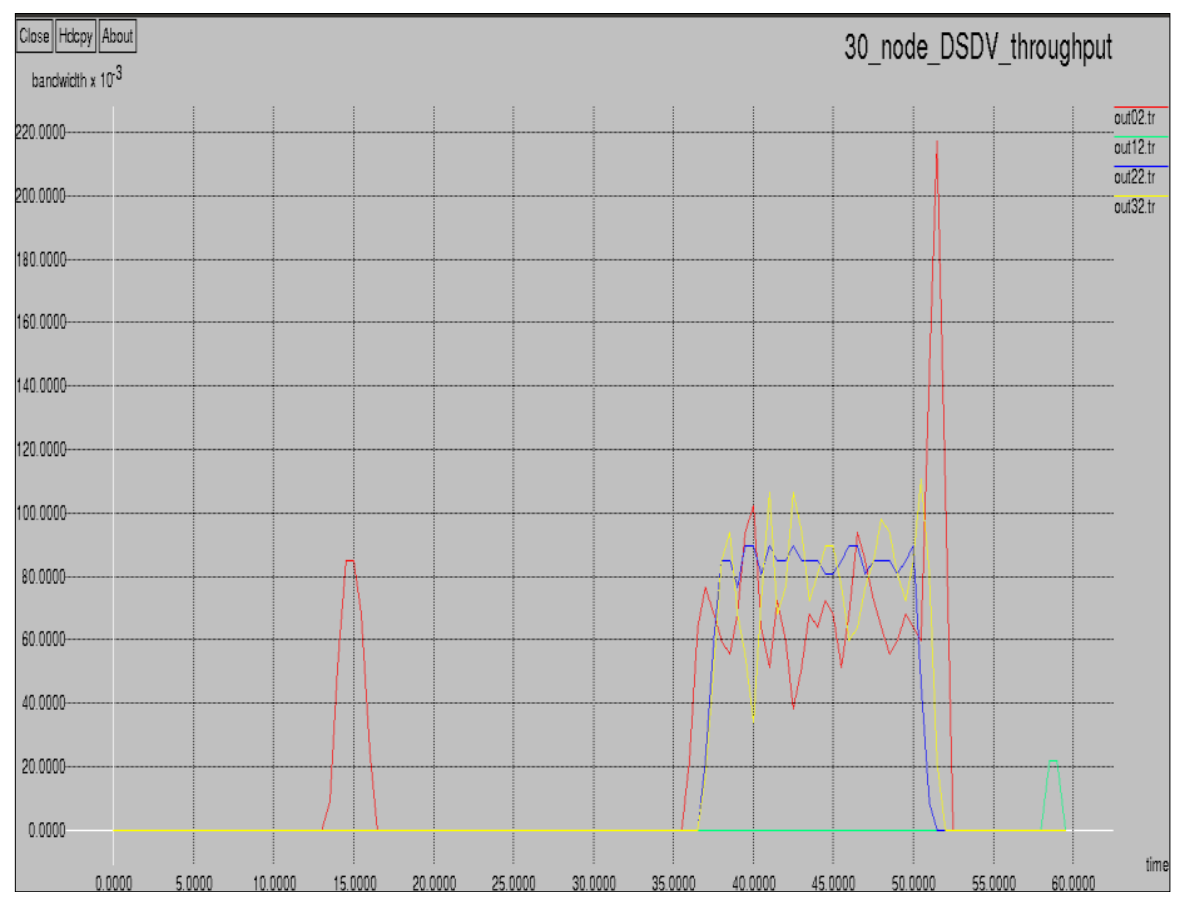

Figure 2. DSDV Throughput

Table 4. DSDV Throughput Comparison Between Three Sources - Sink Nodes

\begin{tabular}{|l|l|l|l|}
\hline Time in sec & N0 - N1 & N5 - N6 & N3 - N6 \\
\hline 13.5 & 0.08 & 0 & 0 \\
\hline 37 & 0.017 & 0.017 & 0.01 \\
\hline 40 & 0.1 & 0.08 & 0.03 \\
\hline 42.5 & 0.03 & 0.1 & 0.1 \\
\hline 43.5 & 0.07 & 0.08 & 0.07 \\
\hline 46 & 0.06 & 0.05 & 0.05 \\
\hline 50.5 & 0.05 & 0.04 & 0.1 \\
\hline 51.5 & 0.2 & 0 & 0.02 \\
\hline
\end{tabular}

Table 4 shows the throughput of DSDV. It shows that a node 0 active at $13.5 \mathrm{sec}$, node 5 and 3 active at $37 \mathrm{sec}$, below this time interval throughput remain zero. The highest value of DSDV throughput at node $0-1$ at $51.5 \mathrm{sec}$ is 0.2 .

3.1.2. Packet Loss: Packet loss is lost of packets during sending and forwarding them in the network. Figure 3 shows graph, in this $x$-axis shows the time and $y$-axis shows the packet loss in $10^{3}$. 


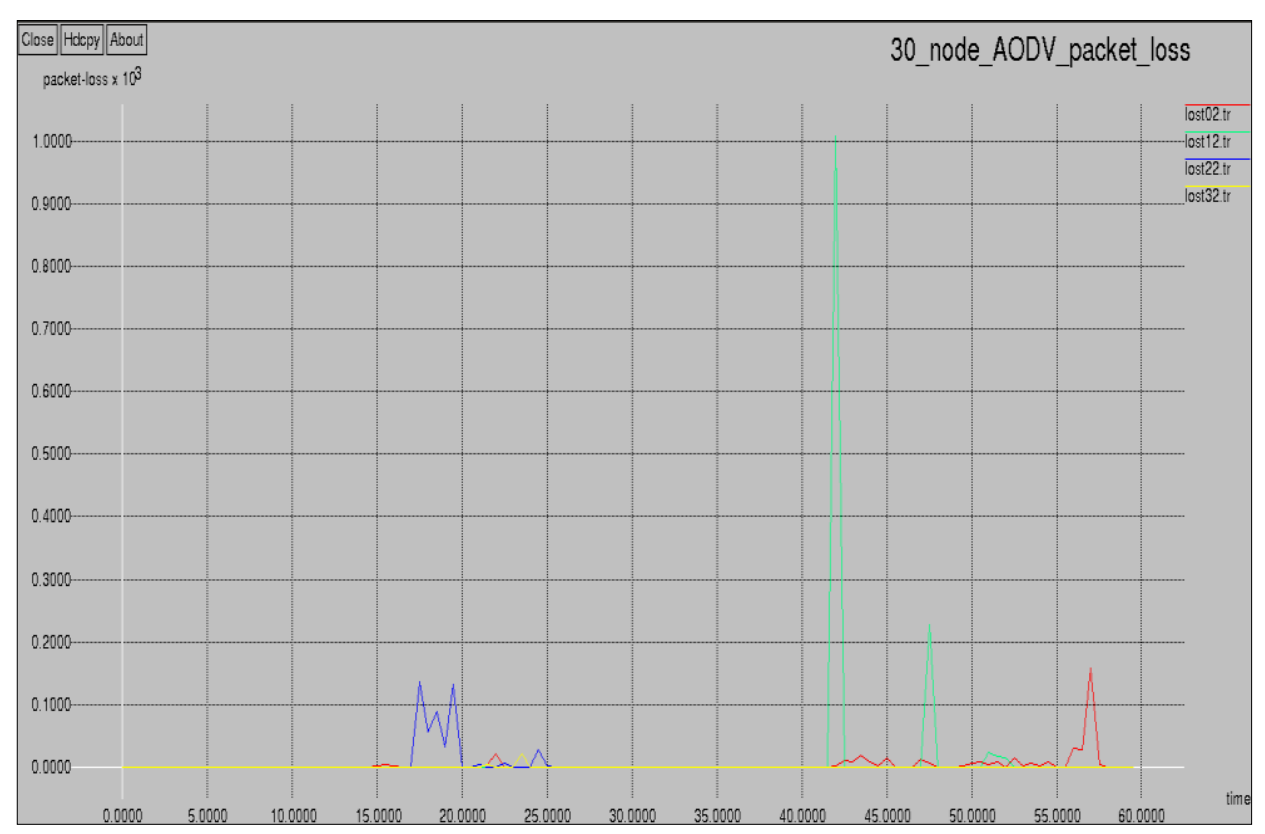

Figure 3. AODV Packet Loss

Table 5. AODV Packet Loss Comparison Between Three Sources - Sink Nodes

\begin{tabular}{|c|c|c|c|}
\hline $\begin{array}{c}\text { Time in } \\
\text { sec }\end{array}$ & N0 - N1 & N5 - N6 & N3 - N6 \\
\hline 15.5 & 4 & 0 & 0 \\
\hline 17.5 & 0 & 136 & 0 \\
\hline 18.5 & 0 & 88 & 0 \\
\hline 19.5 & 0 & 132 & 0 \\
\hline 22 & 22 & 0 & 0 \\
\hline 23.5 & 0 & 0 & 22 \\
\hline 24.5 & 0 & 28 & 0 \\
\hline 43.5 & 20 & 0 & 0 \\
\hline 57 & 158 & 0 & 0 \\
\hline
\end{tabular}

At Table 5 shows that the maximum value of AODV packet loss ratio at $57 \mathrm{sec}$ is 158 at node $0-1$.

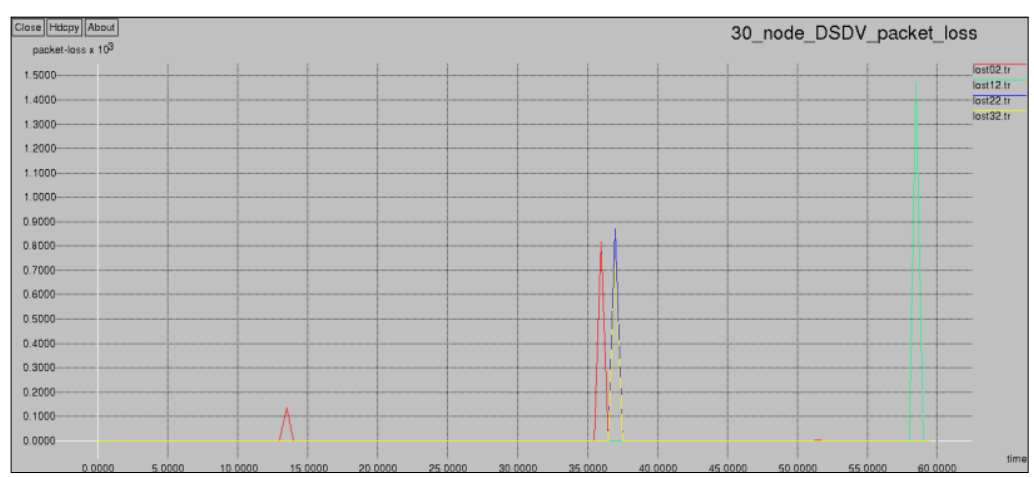

Figure 4. DSDV Packet Loss 
Table 6. DSDV Packet Loss Comparison Between Three Sources - Sink Nodes

\begin{tabular}{|l|l|l|l|}
\hline Time in sec & N0 - N1 & N5 - N6 & N3 - N6 \\
\hline 13.5 & 0 & 0 & 0 \\
\hline 15.5 & 136 & 0 & 0 \\
\hline 36 & 818 & 0 & 0 \\
\hline 37 & 0 & 870 & 750 \\
\hline 51.5 & 2 & 0 & 0 \\
\hline
\end{tabular}

Table 6 shows that the maximum value of DSDV packet loss ratio at node 5-6 at $37 \mathrm{sec}$ is 870 .

3.1.3. Routing delay: Figure 5 shows graph, in this $y$-axis shows the routing delay in sec and $\mathrm{x}$-axis shows the time. It is the time elapses to reach the data packet to the destination. This includes delays caused by retransmission of packets, route finding and queuing packets in the buffer.

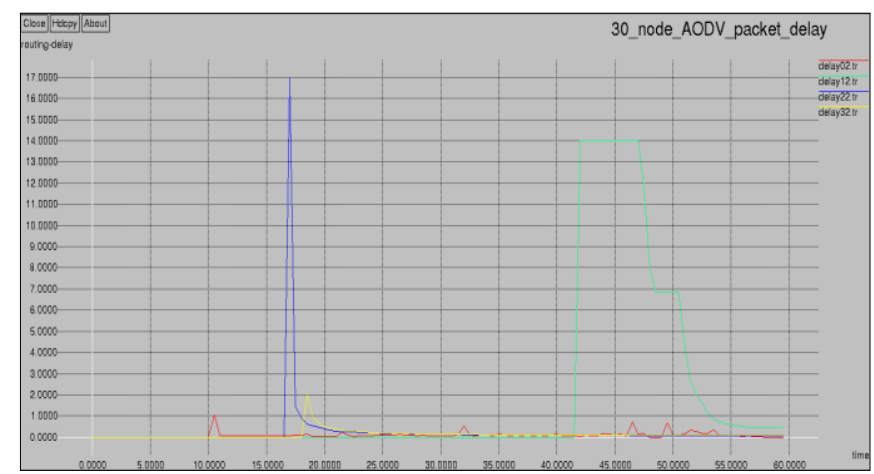

Figure 5. AODV Routing Delay

Table 7. AODV Routing Delay Comparison Between Three Sources - Sink Nodes

\begin{tabular}{|c|c|c|c|}
\hline $\begin{array}{c}\text { Time in } \\
\text { sec }\end{array}$ & N0 - N1 & N5 - N6 & N3 - N6 \\
\hline 5 & 0 & 0 & 0 \\
\hline 10.5 & 1.04 & 0 & 0 \\
\hline 17 & 0.05 & 16.94 & 0 \\
\hline 18.5 & 0.128 & 0.59 & 2.04 \\
\hline 32 & 0.54 & 0.11 & 0.12 \\
\hline 45.5 & 0.13 & 0.08 & 0.08 \\
\hline 46.5 & 0.72 & 0.08 & 0.09 \\
\hline 49.5 & 0.66 & 0.07 & 0.08 \\
\hline 52 & 0.16 & 0.07 & 0.08 \\
\hline
\end{tabular}

Table 7 shows that node $0-1$ starts showing delay at $10.5 \mathrm{sec}$, node $5-6$ starts at 17 sec and node $3-6$ starts at $18.5 \mathrm{sec}$, below these values it remains zero. The maximum value of AODV routing delay at $17 \mathrm{sec}$ is 16.94 at node 5-6. The average end to end delay in the AODV network is $1.73 \mathrm{sec}$ defined in network information. 


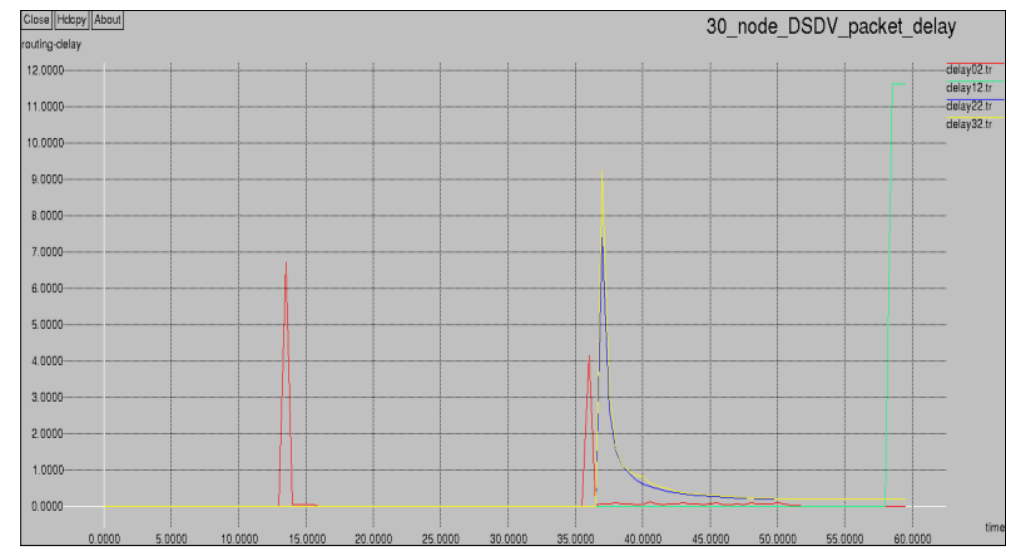

Figure 6. DSDV Routing Delay

Table 8. DSDV Routing Delay Comparison Between Three Sources - Sink Nodes

\begin{tabular}{|c|c|c|c|}
\hline $\begin{array}{c}\text { Time in } \\
\text { sec }\end{array}$ & N0 - N1 & N5 - N6 & N3 - N6 \\
\hline 5 & 0 & 0 & 0 \\
\hline 13.5 & 6.73 & 0 & 0 \\
\hline 36 & 4.14 & 0 & 0 \\
\hline 37 & 0.05 & 7.39 & 9.24 \\
\hline 39.5 & 0.03 & 0.71 & 0.83 \\
\hline 42 & 0.06 & 0.39 & 0.46 \\
\hline 47.5 & 0.05 & 0.2 & 0.24 \\
\hline 51.5 & 0.01 & 0.01 & 0.01 \\
\hline 56 & 0 & 0.18 & 0.19 \\
\hline
\end{tabular}

Table 8 shows that node $0-1$ starts showing delay at $13.5 \mathrm{sec}$, node $5-6,3-6$ starts at $37 \mathrm{sec}$, below these values it remains zero. The maximum value of DSDV routing delay at node $3-6$ at $37 \mathrm{sec}$ is $9.24 \mathrm{sec}$. The average end to end delay in the DSDV network is 0.47 sec defined in network information.

3.1.4. Cumulative Sum of Dropped Packets: The cumulative sum of dropped packets metric is used to find the data packets that are not reached between two nodes.

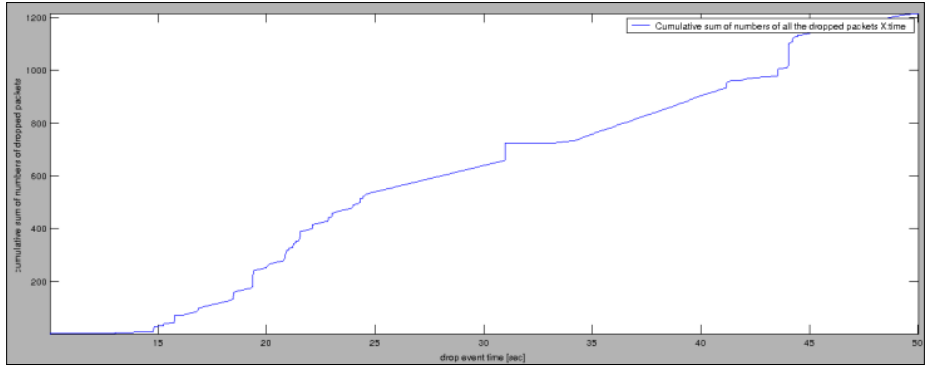

Figure 7. AODV Cumulative Sum of Number of Dropped Packets

Figure 7 shows, the AODV dropped packets. In this graph, the y-axis shows the Cumulative sum of number of dropped packets and x-axis shows the time. At the beginning of the simulation, there was zero dropped packets nearly at $10 \mathrm{sec}$ and after that 
it goes up and ends up to 1216 cumulative sum of dropped packets from the 6416 sent packets.

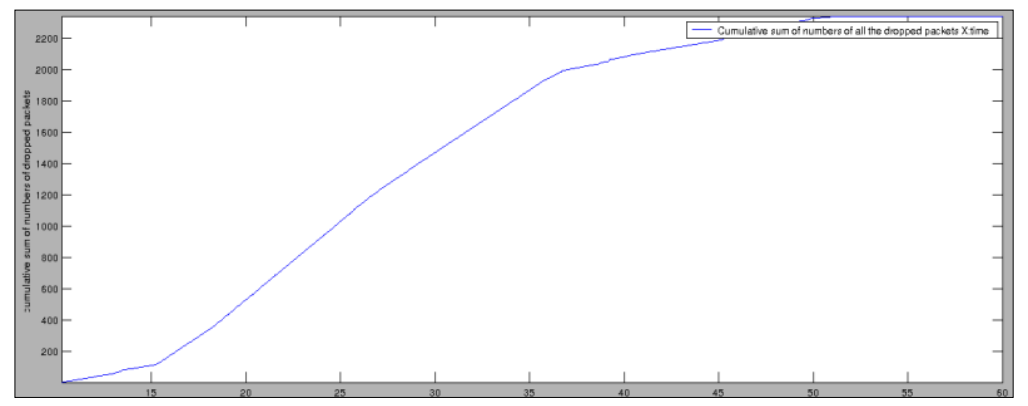

Figure 8. DSDV Cumulative Sum of Number of Dropped Packets

Figure 8 shows, the DSDV dropped packets. At the beginning of the simulation, the dropped packets are increasing and ends up to 2343 cumulative sum of dropped packets from the 3249 sent packets.

3.1.5. Network Information: Trace analyzer file shows the network information in NS2. This helps in comparative study of different parameters.

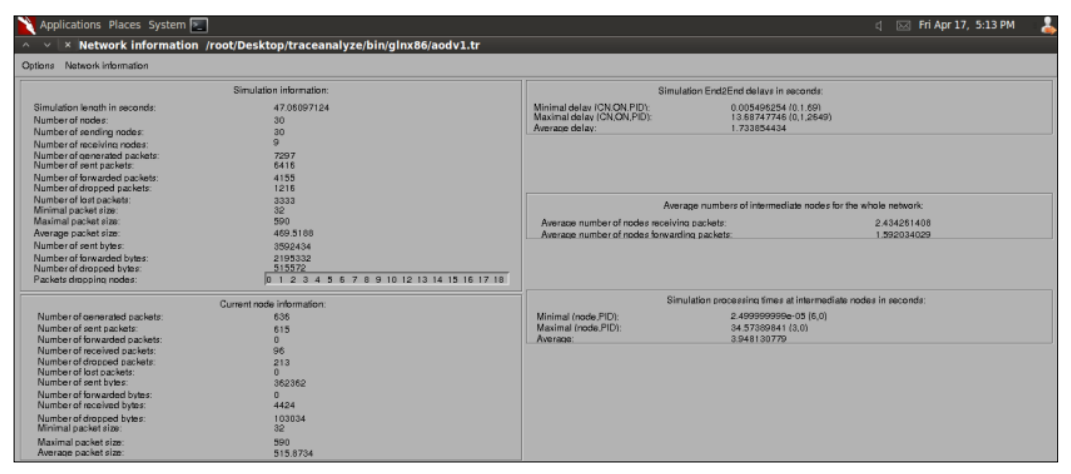

Figure 9. AODV Network Information

Figure 9 shows the network information. It shows that the total no of generating packets in AODV is 7297. Out of this 3249 are sent for transmission. The total number of dropped packets in the network is 1216 and the number of lost packets are 3333 . The total number of forwarded packets is 4155 . The PDF of AODV is $15.6 \%$.

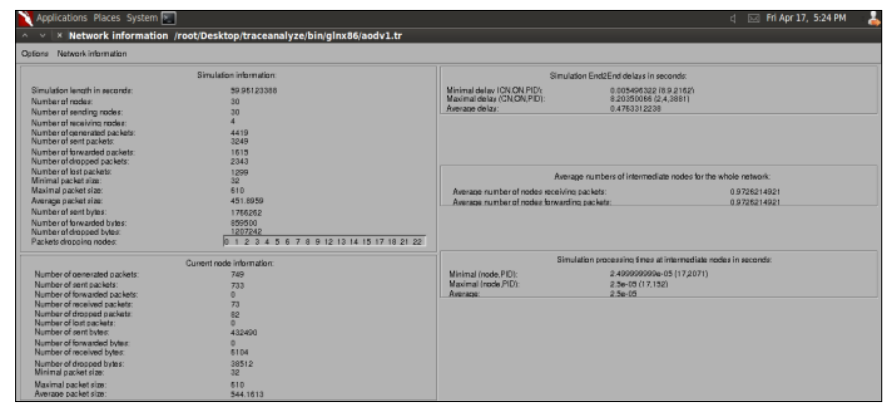

Figure 10. DSDV Network Information 
Figure 10 shows the network information. It shows that total no of generating packets in DSDV are 4419. Out of this 3249 are sent for transmission. The total number of dropped packets in the network is 2343 and the number of lost packets are 1299. The total numbers of forwarded packets are 1615. The PDF of DSDV is $9.95 \%$.

3.1.6. Calculation of Energy Spends for Transmission and Reception of a Single Packet: For the simulations of energy model, the typical transmission power used is 1.4 joules and reception power used is 0.9 joules. The idle state power is 0.73 joules with the minimum packet length of 32 bytes. The total energy consumption in sending and receiving a single packet is defined as:

\section{Consumed energy at each node}

$=$ Packet length $*$ Tx power + Rx power + idle power

Example: Energy consumed at node 0

$=32$ bytes $* 1.4 \mathrm{~J}+0.9 \mathrm{~J}+0.73 \mathrm{~J}$

+ Power spend in control overheads in $\mathrm{J}=$ nearly $50 \mathrm{~J}$

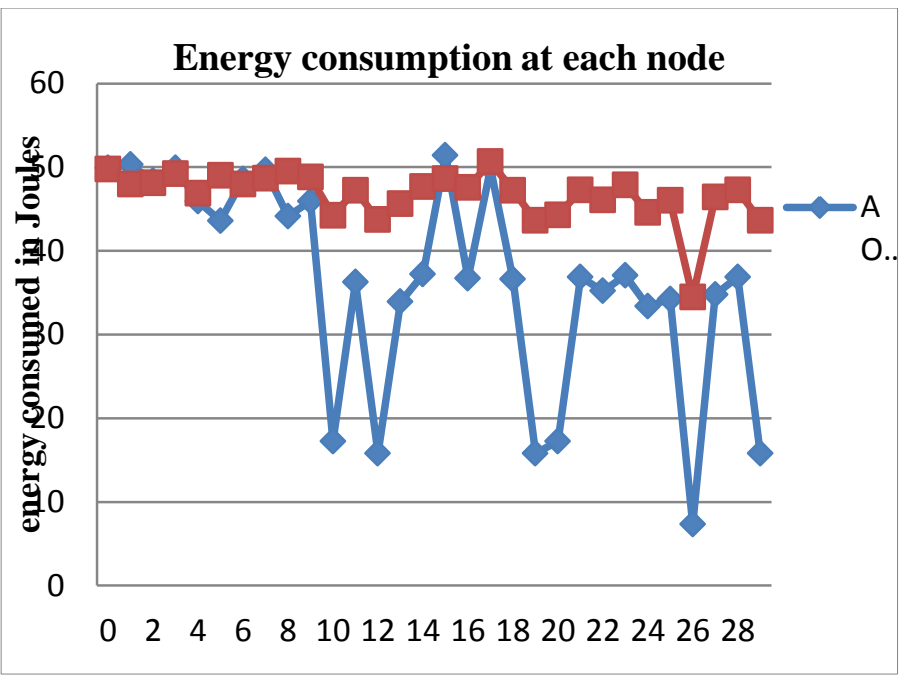

Figure 11. Energy Consumption at Each Node

The figure 11 shows that node 15 has the highest energy consumption nearly $51.4 \mathrm{~J}$ and node 26 has least energy consumption nearly 7.3 J in AODV. The graph also shows that node 17 has highest energy consumption nearly $50.6 \mathrm{~J}$ and node 26 has less nearly $36.4 \mathrm{~J}$ in DSDV. AODV has slightly higher energy consumption than DSDV.

3.1.7. Calculate Residual Energy at each Node: The amount of energy remains in a node after some activity. 


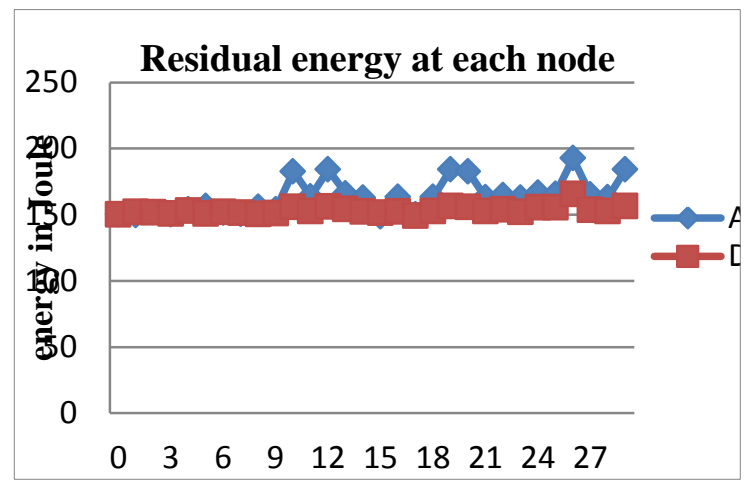

Figure 12. Residual Energy at Each Node

The Figure 12 shows that node 26 has highest remaining energy nearly $192.6 \mathrm{~J}$ and node 15 has least residual energy nearly 148.5 in AODV. The graph also shows that node 26 has highest remaining energy nearly $165.5 \mathrm{~J}$ and node 17 has least remaining energy nearly 149.3 in DSDV. AODV has higher residual energy than DSDV.

In AODV, the total energy consumption of all nodes is $1295.74 \mathrm{~J}$. The average energy consumption (AEC) by nodes in the network is $43.1912 \mathrm{~J}$. In DSDV, the total energy consumption of all nodes is $1599.72 \mathrm{~J}$. The average energy consumption (AEC) by nodes in the network is $53.3241 \mathrm{~J}$

\section{Comparison of Proposed Routing Protocols}

After simulations performed in NS2, results are shown in tabular form which compares performance parameters of AODV, DSDV routing protocol and is shown below:

Table 9. Comparison of Performance Parameters of AODV and DSDV at a Node

\begin{tabular}{|c|c|c|}
\hline Parameters & AODV & DSDV \\
\hline $\begin{array}{c}\text { No of generating } \\
\text { packets }\end{array}$ & 636 & 749 \\
\hline Packet sent & 615 & 733 \\
\hline Packet received & 96 & 73 \\
\hline $\begin{array}{c}\text { Packet Delivery } \\
\text { Ratio }\end{array}$ & $15 \%$ & $9.74 \%$ \\
\hline $\begin{array}{c}\text { No. of Dropped } \\
\text { Packets }\end{array}$ & 213 & 82 \\
\hline Throughput & 0.21 & 0.21 \\
\hline Simulation End time & $47.0 \mathrm{sec}$ & $59.96 \mathrm{sec}$ \\
\hline $\begin{array}{c}\text { Average no of } \\
\text { receiving packets at } \\
\text { Intermediate nodes }\end{array}$ & 2.436 & 0.972 \\
\hline $\begin{array}{c}\text { Average no of } \\
\text { forwarding packets at } \\
\text { intermediate nodes }\end{array}$ & 1.592 & 0.972 \\
\hline $\begin{array}{c}\text { Average Energy } \\
\text { Consumption }\end{array}$ & $43.19 \mathrm{~J}$ & $53.32 \mathrm{~J}$ \\
\hline $\begin{array}{c}\text { Average End to End } \\
\text { delay }\end{array}$ & $1.733 \mathrm{sec}$ & $0.476 \mathrm{sec}$ \\
\hline
\end{tabular}




\section{Conclusion and Future Scope}

This paper presents the simulation result of on-demand AODV and table driven DSDV routing protocol on different performance metrics, i.e. throughput, packet loss, end to end delay, cumulative sum of dropped packets and calculation of average energy consumption. The results obtained from the simulation in NS2 reveals that AODV is much better than DSDV.

With respect to the packet delivery ratio, packet loss, no of dropped packets, average no of receiving packets at intermediate nodes, average no of forwarding packets, the average energy consumption of AODV is more than DSDV. DSDV performs well as having a low average end to end delay.

For future research similar routing protocol can be implemented on a variable number of nodes, packet size, speed of a node, pause time, simulation time, and number of connections. The performance of the network can also evaluate using new routing protocol.

\section{Acknowledgment}

The author wishes to thank Ms. Nitika Chowdhary for the guidance provided in this research work.

\section{References}

[1] S. S. Getsy, S. P. Neelavathy and D. Sridharan, "Evaluation and Comparison of Emerging Energy Efficient Routing Protocols in Manet", vol. 01, (2010).

[2] K. Prabu and Dr. A. Subramani, "Performance Comparison of Routing Protocols in MANET", International Journal of Advanced Research in Computer Science and Software Engineering, 2277 128X, (2012).

[3] E. Pragati and Dr. R. Nath, "Performance Evaluation of AODV, LEACH \& TORA Protocols through Simulation", International Journal of Advanced Research in Computer Science and Software Engineering, vol. 2277, no. 128X, (2012).

[4] T. Nema, A. Waoo, P. S. Patheja and PH. D. S. Sharma, "Energy based AODV Routing Algorithm with Sleep Mode in MANETs", vol. 58, (2012).

[5] E. Pragati and Dr. R. Nath, "Performance Evaluation of AODV, LEACH \& TORA Protocols through Simulation", vol. 2, no. 7, (2014) July.

[6] M. V. Khiavi and S. Jamali, "Performance Comparison of AODV, DSDV, DSR and TORA Routing Protocols in MANETs", vol. 3, no. 7, (2012).

[7] R. R. Gupta, Mahendra and K. Mishra, "Power Saving Routing Protocol for Ad hoc Networks based on AODV", International Journal of Computer Applications, vol. 85, no. 19, (2014)January.

[8] M. Rafiq and M. Kumar, "J. Comparative Analysis of Energy Efficient Routing Protocols in MANET", International Journal of Computer Applications, vol. 82, no. 5, (2013)November.

[9] P. Manickam, T. Guru Baskar, M.Girija and Dr.D.Manimegala, "Performance Comparisons of Routing Protocols in Mobile Ad Hoc Network", International Journal of Wireless \& Mobile Networks (IJWMN), vol. 3, no. 1, (2011) February.

\section{Authors}

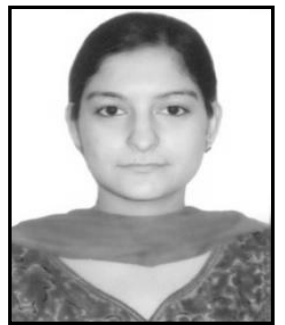

Anmolpreet Kaur, (B. Tech) received the bachelor's degree in the Electronics and Communication, in 2014 from Punjab Technical University, India. She is a student of the M. Tech in Electronics and Communication Engineering department at Guru Nanak Dev University Regional Campus Jalandhar (INDIA). Her research area of interest is MANET. 


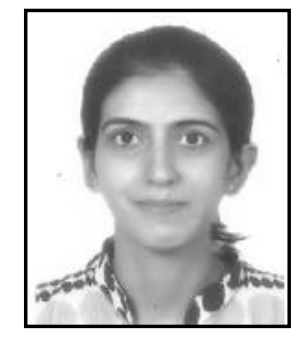

Nitika Chowdhary, (B. Tech, M. Tech) received the master's degree in computer science and engineering, in 2013 and the bachelor's degree in the information technology, in 2011 from Punjab Technical University, India. She is currently working as Assistant Professor in Computer Science and Engineering department at Guru Nanak Dev University Regional Campus Jalandhar. Her research interests include security, distributed networks, and cloud computing.

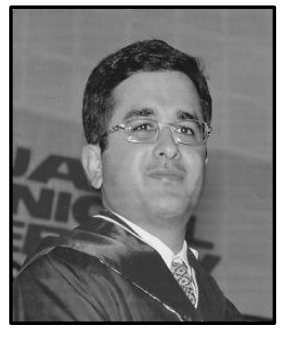

Jyoteesh Malhotra, (B. Tech, M. Tech, PhD) is involved in teaching and research in Electronics and Communication Department at Guru Nanak Dev University Regional Campus Jalandhar, India. His research area of interest includes statistical modeling of fading channels, fading mitigation techniques in wireless communication, and Wireless BAN. Dr. Malhotra has more than 100 research publication and authored two books. He is a life member of ISTE and editorial board member of many international journal of repute. 